Korean J. Math. 20 (2012), No. 1, pp. 33-46

\title{
ADDITIVE-QUARTIC FUNCTIONAL EQUATION IN NON-ARCHIMEDEAN ORTHOGONALITY SPACES
}

\author{
Hyunju Lee*, Seon Woo Kim, Bum Joon Son, Dong Hwan \\ LeE AND Seung YeOn Kang
}

\begin{abstract}
Using the direct method, we prove the Hyers-Ulam stability of the orthogonally additive-quartic functional equation

$$
f(2 x+y)+f(2 x-y)=4 f(x+y)+4 f(x-y)
$$$$
\text { (0.1) }+10 f(x)+14 f(-x)-3 f(y)-3 f(-y)
$$

for all $x, y$ with $x \perp y$, in non-Archimedean Banach spaces. Here $\perp$ is the orthogonality in the sense of Rätz.
\end{abstract}

\section{Introduction and preliminaries}

In 1897, Hensel [15] introduced a normed space which does not have the Archimedean property. It turned out that non-Archimedean spaces have many nice applications (see [9, 20, 21, 27]).

A valuation is a function | - | from a field $K$ into $[0, \infty)$ such that 0 is the unique element having the 0 valuation, $|r s|=|r| \cdot|s|$ and the triangle inequality holds, i.e.,

$$
|r+s| \leq|r|+|s|, \quad \forall r, s \in K
$$

A field $K$ is called a valued field if $K$ carries a valuation. Throughout this paper, we assume that the base field is a valued field, hence call it simply a field. The usual absolute values of $\mathbb{R}$ and $\mathbb{C}$ are examples of valuations.

Received December 16, 2011. Revised January 11, 2012. Accepted January 15, 2012.

2010 Mathematics Subject Classification: 39B55, 46S10, 39B72, 39B52, 54E40, 47S10, 26E30, 12J25, 46H25.

Key words and phrases: Hyers-Ulam stability, orthogonally additive-quartic functional equation, non-Archimedean normed space, orthogonality space.

${ }^{*}$ Corresponding author. 
Let us consider a valuation which satisfies a stronger condition than the triangle inequality. If the triangle inequality is replaced by

$$
|r+s| \leq \max \{|r|,|s|\}, \quad \forall r, s \in K,
$$

then the function $|\cdot|$ is called a non-Archimedean valuation, and the field is called a non-Archimedean field. Clearly $|1|=|-1|=1$ and $|n| \leq 1$ for all $n \in \mathbb{N}$. A trivial example of a non-Archimedean valuation is the function $|\cdot|$ taking everything except for 0 into 1 and $|0|=0$.

DeFinition 1.1. ([26]) Let $X$ be a vector space over a field $K$ with a non-Archimedean valuation | | |. A function $\|\cdot\|: X \rightarrow[0, \infty)$ is said to be a non-Archimedean norm if it satisfies the following conditions:

(i) $\|x\|=0$ if and only if $x=0$;

(ii) $\|r x\|=|r|\|x\| \quad(r \in K, x \in X)$;

(iii) the strong triangle inequality

$$
\|x+y\| \leq \max \{\|x\|,\|y\|\}, \quad \forall x, y \in X
$$

holds. Then $(X,\|\cdot\|)$ is called a non-Archimedean normed space.

DEFINITION 1.2. (i) Let $\left\{x_{n}\right\}$ be a sequence in a non-Archimedean normed space $X$. Then the sequence $\left\{x_{n}\right\}$ is called Cauchy if for a given $\varepsilon>0$ there is a positive integer $N$ such that

$$
\left\|x_{n}-x_{m}\right\| \leq \varepsilon
$$

for all $n, m \geq N$.

(ii) Let $\left\{x_{n}\right\}$ be a sequence in a non-Archimedean normed space $X$. Then the sequence $\left\{x_{n}\right\}$ is called convergent if for a given $\varepsilon>0$ there are a positive integer $N$ and an $x \in X$ such that

$$
\left\|x_{n}-x\right\| \leq \varepsilon
$$

for all $n \geq N$. Then we call $x \in X$ a limit of the sequence $\left\{x_{n}\right\}$, and denote by $\lim _{n \rightarrow \infty} x_{n}=x$.

(iii) If every Cauchy sequence in $X$ converges, then the non-Archimedean normed space $X$ is called a non-Archimedean Banach space.

Assume that $\mathrm{X}$ is a real inner product space and $f: X \rightarrow \mathbb{R}$ is a solution of the orthogonal Cauchy functional equation $f(x+y)=$ $f(x)+f(y),\langle x, y\rangle=0$. By the Pythagorean theorem $f(x)=\|x\|^{2}$ is a solution of the Cauchy functional equation. Of course, this function does not satisfy the additivity equation everywhere. Thus orthogonal Cauchy 
functional equation is not equivalent to the classic Cauchy functional equation on the whole inner product space.

G. Pinsker [30] characterized orthogonally additive functionals on an inner product space when the orthogonality is the ordinary one in such spaces. K. Sundaresan [39] generalized this result to arbitrary Banach spaces equipped with the Birkhoff-James orthogonality. The orthogonal Cauchy functional equation

$$
f(x+y)=f(x)+f(y), \quad x \perp y,
$$

in which $\perp$ is an abstract orthogonality relation, was first investigated by S. Gudder and D. Strawther [14]. They defined $\perp$ by a system consisting of five axioms and described the general semi-continuous real-valued solution of conditional Cauchy functional equation. In 1985, J. Rätz [36] introduced a new definition of orthogonality by using more restrictive axioms than of S. Gudder and D. Strawther. Moreover, he investigated the structure of orthogonally additive mappings. J. Rätz and Gy. Szabó [37] investigated the problem in a rather more general framework.

Let us recall the orthogonality in the sense of J. Rätz ; cf. [36].

Suppose $X$ is a real vector space with $\operatorname{dim} X \geq 2$ and $\perp$ is a binary relation on $X$ with the following properties:

$\left(O_{1}\right)$ totality of $\perp$ for zero: $x \perp 0,0 \perp x$ for all $x \in X$;

$\left(O_{2}\right)$ independence: if $x, y \in X-\{0\}, x \perp y$, then $x, y$ are linearly independent;

$\left(O_{3}\right)$ homogeneity: if $x, y \in X, x \perp y$, then $\alpha x \perp \beta y$ for all $\alpha, \beta \in \mathbb{R}$;

$\left(O_{4}\right)$ the Thalesian property: if $P$ is a 2-dimensional subspace of $X, x \in$ $P$ and $\lambda \in \mathbb{R}_{+}$, which is the set of nonnegative real numbers, then there exists $y_{0} \in P$ such that $x \perp y_{0}$ and $x+y_{0} \perp \lambda x-y_{0}$.

The pair $(X, \perp)$ is called an orthogonality space. By an orthogonality normed space we mean an orthogonality space having a normed structure.

Some interesting examples are

(i) The trivial orthogonality on a vector space $X$ defined by $\left(O_{1}\right)$, and for non-zero elements $x, y \in X, x \perp y$ if and only if $x, y$ are linearly independent.

(ii) The ordinary orthogonality on an inner product space $(X,\langle.,\rangle$.$) given$ by $x \perp y$ if and only if $\langle x, y\rangle=0$.

(iii) The Birkhoff-James orthogonality on a normed space $(X,\|\|$.$) de-$ fined by $x \perp y$ if and only if $\|x+\lambda y\| \geq\|x\|$ for all $\lambda \in \mathbb{R}$. 
The relation $\perp$ is called symmetric if $x \perp y$ implies that $y \perp x$ for all $x, y \in X$. Clearly examples (i) and (ii) are symmetric but example (iii) is not. It is remarkable to note, however, that a real normed space of dimension greater than 2 is an inner product space if and only if the Birkhoff-James orthogonality is symmetric. There are several orthogonality notions on a real normed space such as Birkhoff-James, Boussouis, Singer, Carlsson, unitary-Boussouis, Roberts, Pythagorean, isosceles and Diminnie (see [1]-[4], [10, 18]).

The stability problem of functional equations originated from the following question of Ulam [41]: Under what condition does there exist an additive mapping near an approximately additive mapping? In 1941, Hyers [16] gave a partial affirmative answer to the question of Ulam in the context of Banach spaces. In 1978, Th.M. Rassias [31] extended the theorem of Hyers by considering the unbounded Cauchy difference.

In [22], Lee et al. considered the following quartic functional equation

$$
f(2 x+y)+f(2 x-y)=4 f(x+y)+4 f(x-y)+24 f(x)-6 f(y) .
$$

It is easy to show that the function $f(x)=x^{4}$ satisfies the functional equation (1.1), which is called a quartic functional equation and every solution of the quartic functional equation is said to be a quartic mapping.

R. Ger and J. Sikorska [13] investigated the orthogonal stability of the Cauchy functional equation $f(x+y)=f(x)+f(y)$, namely, they showed that if $f$ is a mapping from an orthogonality space $X$ into a real Banach space $Y$ and $\|f(x+y)-f(x)-f(y)\| \leq \varepsilon$ for all $x, y \in X$ with $x \perp y$ and some $\varepsilon>0$, then there exists exactly one orthogonally additive mapping $g: X \rightarrow Y$ such that $\|f(x)-g(x)\| \leq \frac{16}{3} \varepsilon$ for all $x \in X$.

The first author treating the stability of the quadratic equation was F. Skof [38] by proving that if $f$ is a mapping from a normed space $X$ into a Banach space $Y$ satisfying $\| f(x+y)+f(x-y)-2 f(x)-$ $2 f(y) \| \leq \varepsilon$ for some $\varepsilon>0$, then there is a unique quadratic mapping $g: X \rightarrow Y$ such that $\|f(x)-g(x)\| \leq \frac{\varepsilon}{2}$. P.W. Cholewa [5] extended the Skof's theorem by replacing $X$ by an abelian group $G$. The Skof's result was later generalized by S. Czerwik [6] in the spirit of Hyers-UlamRassias. During the last decades several stability problems of functional equations have been investigated in the spirit of Hyers-Ulam-Rassias (see $[7,8,17,19,29],[32]-[35])$. 
The orthogonally quadratic equation

$$
f(x+y)+f(x-y)=2 f(x)+2 f(y), x \perp y
$$

was first investigated by F. Vajzovic [42] when $X$ is a Hilbert space, $Y$ is the scalar field, $f$ is continuous and $\perp$ means the Hilbert space orthogonality. Later, H. Drljević [11], M. Fochi [12], M.S. Moslehian $[23,24]$ and Gy. Szabó [40] generalized this result. See also [25, 28].

This paper is organized as follows: In Section 2, we prove the HyersUlam stability of the orthogonally additive-quartic functional equation (0.1) in non-Archimedean orthogonality spaces for an odd mapping.

In Section 3, we prove the Hyers-Ulam stability of the orthogonally additive-quartic functional equation (0.1) in non-Archimedean orthogonality spaces for an even mapping.

Throughout this paper, assume that $(X, \perp)$ is a non-Archimedean orthogonality space and that $(Y,\|\cdot\|)$ is a real non-Archimedean Banach space. Assume that $|2| \neq 1$.

\section{Stability of the orthogonally additive-quartic functional equation: an odd mapping case}

In this section, we deal with the stability problem for the orthogonally additive-quartic functional equation

$$
\begin{aligned}
D f(x, y): & =f(2 x+y)+f(2 x-y)-4 f(x+y)-4 f(x-y) \\
& -10 f(x)-14 f(-x)+3 f(y)+3 f(-y)=0
\end{aligned}
$$

for all $x, y \in X$ with $x \perp y$ in non-Archimedean Banach spaces: an odd mapping case.

Definition 2.1. An odd mapping $f: X \rightarrow Y$ is called an orthogonally additive mapping if

$$
f(2 x+y)+f(2 x-y)=4 f(x+y)+4 f(x-y)-4 f(x)
$$

for all $x, y \in X$ with $x \perp y$.

Theorem 2.2. Let $\varphi: X^{2} \rightarrow[0, \infty)$ be a function such that

$$
\Phi(x, y):=\sum_{i=0}^{\infty} \frac{1}{|2|^{i}} \varphi\left(2^{i} x, 2^{i} y\right)<+\infty
$$


for all $x, y \in X$ with $x \perp y$. Let $f: X \rightarrow Y$ be an odd mapping satisfying

$$
\|D f(x, y)\| \leq \varphi(x, y)
$$

for all $x, y \in X$ with $x \perp y$. Then there exists a unique orthogonally additive mapping $A: X \rightarrow Y$ such that

$$
\|f(x)-A(x)\| \leq \frac{1}{|2|^{2}} \Phi(x, 0)
$$

for all $x \in X$.

Proof. Putting $y=0$ in (2.1), we get

$$
\|2 f(2 x)-4 f(x)\| \leq \varphi(x, 0)
$$

for all $x \in X$, since $x \perp 0$. So

$$
\left\|\frac{1}{2} f(2 x)-f(x)\right\| \leq \frac{1}{|2|^{2}} \varphi(x, 0)
$$

for all $x \in X$. Replacing $x$ by $2^{n} x$ in (2.3), we get

$$
\left\|\frac{1}{2} f\left(2^{n+1} x\right)-f\left(2^{n} x\right)\right\| \leq \frac{1}{|2|^{2}} \varphi\left(2^{n} x, 0\right)
$$

for all $n \geq 0$ and all $x \in X$, since $2^{n} x \perp 0$. So

$$
\left\|\frac{1}{2^{n+1}} f\left(2^{n+1} x\right)-\frac{1}{2^{n}} f\left(2^{n} x\right)\right\| \leq \frac{1}{|2|^{n+2}} \varphi\left(2^{n} x, 0\right)
$$

for all $n \geq 0$ and all $x \in X$.

Now we define a mapping $g$ such that

$$
g(n, x):=\frac{1}{2^{n+1}} f\left(2^{n+1} x\right)-\frac{1}{2^{n}} f\left(2^{n} x\right)
$$

for all $n \geq 0$ and all $x \in X$. Then

$$
\|g(n, x)\| \leq \frac{1}{|2|^{n+2}} \varphi\left(2^{n} x, 0\right)
$$


for all $n \geq 0$ and all $x \in X$. So

$$
\begin{aligned}
\left\|\frac{1}{2^{m}} f\left(2^{m} x\right)-\frac{1}{2^{n}} f\left(2^{n} x\right)\right\| & =\left\|\sum_{i=n}^{m-1} g(i, x)\right\| \\
& \leq \max \{\|g(n, x)\|, \cdots,\|g(m-1, x)\|\} \\
& \leq \sum_{i=n}^{m-1}\|g(i, x)\| \\
& \leq \sum_{i=n}^{m-1} \frac{1}{|2|^{i+2}} \varphi\left(2^{i} x, 0\right),
\end{aligned}
$$

which tends to zero as $n \rightarrow \infty$, for all $m>n \geq 0$ and all $x \in X$. Thus the sequence $\left\{\frac{1}{2^{n}} f\left(2^{n} x\right)\right\}$ is a Cauchy sequence. Since $Y$ is a nonArchimedean Banach space, The sequence $\left\{\frac{1}{2^{n}} f\left(2^{n} x\right)\right\}$ converges. So we can define a mapping $A: X \rightarrow Y$ such that

$$
\lim _{n \rightarrow \infty} \frac{1}{2^{n}} f\left(2^{n} x\right)=A(x)
$$

for all $x \in X$.

Replacing $x, y$ by $2^{n} x, 2^{n} y$ in (2.1), respectively, we get

$$
\left\|D f\left(2^{n} x, 2^{n} y\right)\right\| \leq \varphi\left(2^{n} x, 2^{n} y\right)
$$

for all $x, y \in X$ with $x \perp y$, since $2^{n} x \perp 2^{n} y$. Then

$$
\left\|\frac{1}{2^{n}} D f\left(2^{n} x, 2^{n} y\right)\right\| \leq \frac{1}{|2|^{n}} \varphi\left(2^{n} x, 2^{n} y\right)
$$

for all $x, y \in X$ with $x \perp y$. So

$$
\|D A(x, y)\|=\lim _{n \rightarrow \infty}\left\|\frac{1}{2^{n}} D f\left(2^{n} x, 2^{n} y\right)\right\| \leq \lim _{n \rightarrow \infty} \frac{1}{|2|^{n}} \varphi\left(2^{n} x, 2^{n} y\right)=0
$$

for all $x, y \in X$ with $x \perp y$. Thus

$$
D A(x, y)=0
$$

for all $x, y \in X$ with $x \perp y$.

Since $f(x)$ is an odd mapping, $A(x)$ is an odd mapping. So the mapping $A: X \rightarrow Y$ is an orthogonally additive mapping.

Letting $n=0$ and $m \rightarrow \infty$ in (2.4), we get the inequality (2.2). 
To prove the uniqueness of $A$, let $L: X \rightarrow Y$ be another orthogonally additive mapping satisfying (2.2).

$$
\begin{aligned}
& \|A(x)-L(x)\| \\
& \leq\left\|\frac{1}{2^{n}} A\left(2^{n} x\right)-\frac{1}{2^{n}} L\left(2^{n} x\right)\right\| \\
& \leq \max \left\{\left\|\frac{1}{2^{n}} A\left(2^{n} x\right)-\frac{1}{2^{n}} f\left(2^{n} x\right)\right\|,\left\|\frac{1}{2^{n}} f\left(2^{n} x\right)-\frac{1}{2^{n}} L\left(2^{n} x\right)\right\|\right\} \\
& \leq \frac{1}{|2|^{n+2}} \Phi\left(2^{n} x, 0\right),
\end{aligned}
$$

which tends to zero as $n \rightarrow \infty$. So $A: X \rightarrow Y$ is unique.

Therefore, there exists a unique orthogonally additive mapping $A$ : $X \rightarrow Y$ satisfying (2.2). This completes the proof.

Corollary 2.3. Let $p>1$. Let $f: X \rightarrow Y$ be an odd mapping satisfying

$$
\|D f(x, y)\| \leq \theta\left(\|x\|^{p}+\|y\|^{p}\right)
$$

for all $x, y \in X$ with $x \perp y$. Then there exists a unique orthogonally additive mapping $A: X \rightarrow Y$ such that

$$
\|f(x)-A(x)\| \leq \frac{\theta}{|2|\left(|2|-|2|^{p}\right)}\|x\|^{p}
$$

for all $x \in X$.

Theorem 2.4. Let $\varphi: X^{2} \rightarrow[0, \infty)$ be a function such that

$$
\Phi(x, y):=\sum_{i=1}^{\infty}|2|^{i} \varphi\left(\frac{x}{2^{i}}, \frac{y}{2^{i}}\right)<+\infty
$$

for all $x, y \in X$ with $x \perp y$. Let $f: X \rightarrow Y$ be an odd mapping satisfying (2.1). Then there exists a unique orthogonally additive mapping $A$ : $X \rightarrow Y$ such that

$$
\|f(x)-A(x)\| \leq \frac{1}{|2|^{2}} \Phi(x, 0)
$$

for all $x \in X$.

Proof. It follows from (2.3) that

$$
\left\|f(x)-2 f\left(\frac{x}{2}\right)\right\| \leq \frac{1}{|2|} \varphi\left(\frac{x}{2}, 0\right)
$$


for all $x \in X$, since $x \perp 0$.

The rest of the proof is similar to the proof of Theorem 2.2.

Corollary 2.5. Let $0<p<1$. Let $f: X \rightarrow Y$ be an odd mapping satisfying (2.5). Then there exists a unique orthogonally additive mapping $A: X \rightarrow Y$ such that

$$
\|f(x)-A(x)\| \leq \frac{\theta}{|2|\left(|2|^{p}-|2|\right)}\|x\|^{p}
$$

for all $x \in X$.

\section{Stability of the orthogonally additive-quartic functional equation: an even mapping case}

In this section, we deal with the stability problem for the orthogonally additive-quartic functional equation $D f(x, y)=0$ given in the previous section: an even mapping case.

DeFinition 3.1. An even mapping $f: X \rightarrow Y$ is called an orthogonally quartic mapping if

$$
f(2 x+y)+f(2 x-y)=4 f(x+y)+4 f(x-y)+24 f(x)-6 f(y)
$$

for all $x, y \in X$ with $x \perp y$.

Theorem 3.2. Let $\varphi: X^{2} \rightarrow[0, \infty)$ be a function such that

$$
\Psi(x, y):=\sum_{i=0}^{\infty} \frac{1}{|2|^{4 i}} \varphi\left(2^{i} x, 2^{i} y\right)<+\infty
$$

for all $x, y \in X$ with $x \perp y$. Let $f: X \rightarrow Y$ be an even mapping satisfying $f(0)=0$ and (2.1). Then there exists a unique orthogonally quartic mapping $Q: X \rightarrow Y$ such that

$$
\|f(x)-Q(x)\| \leq \frac{1}{|2|^{5}} \Psi(x, 0)
$$

for all $x \in X$.

Proof. Putting $y=0$ in (2.1), we get

$$
\|2 f(2 x)-32 f(x)\| \leq \varphi(x, 0)
$$


for all $x \in X$, since $x \perp 0$. So

$$
\left\|\frac{1}{16} f(2 x)-f(x)\right\| \leq \frac{1}{|2|^{5}} \varphi(x, 0)
$$

for all $x \in X$.

The rest of the proof is similar to the proof of Theorem 2.2.

Corollary 3.3. Let $p>4$. Let $f: X \rightarrow Y$ be an even mapping satisfying $f(0)=0$ and (2.5). Then there exists a unique orthogonally quartic mapping $Q: X \rightarrow Y$ such that

$$
\|f(x)-Q(x)\| \leq \frac{\theta}{|2|\left(|2|^{4}-|2|^{p}\right)}\|x\|^{p}
$$

for all $x \in X$.

TheOREM 3.4. Let $\varphi: X^{2} \rightarrow[0, \infty)$ be a function such that

$$
\Psi(x, y):=\sum_{i=1}^{\infty}|2|^{4 i} \varphi\left(\frac{x}{2^{i}}, \frac{y}{2^{i}}\right)<+\infty
$$

for all $x, y \in X$ with $x \perp y$. Let $f: X \rightarrow Y$ be an even mapping satisfying $f(0)=0$ and (2.1). Then there exists a unique orthogonally quartic mapping $Q: X \rightarrow Y$ such that

$$
\|f(x)-Q(x)\| \leq \frac{1}{|2|^{5}} \Psi(x, 0)
$$

for all $x \in X$.

Proof. It follows from (3.1) that

$$
\left\|f(x)-16 f\left(\frac{x}{2}\right)\right\| \leq \frac{1}{|2|} \varphi\left(\frac{x}{2}, 0\right)
$$

for all $x \in X$, since $x \perp 0$.

The rest of the proof is similar to the proof of Theorem 2.2.

Corollary 3.5. Let $0<p<4$. Let $f: X \rightarrow Y$ be an even mapping satisfying $f(0)=0$ and (2.5). Then there exists a unique orthogonally quartic mapping $Q: X \rightarrow Y$ such that

$$
\|f(x)-Q(x)\| \leq \frac{\theta}{|2|\left(|2|^{p}-|2|^{4}\right)}\|x\|^{p}
$$

for all $x \in X$. 
Let $f_{o}(x)=\frac{f(x)-f(-x)}{2}$ and $f_{e}(x)=\frac{f(x)+f(-x)}{2}$. Then $f_{o}$ is an odd mapping and $f_{e}$ is an even mapping such that $f=f_{o}+f_{e}$.

The above corollaries can be summarized as follows:

Theorem 3.6. Let $p>4$. Let $f: X \rightarrow Y$ be a mapping satisfying $f(0)=0$ and (2.5). Then there exist an orthogonally additive mapping $A: X \rightarrow Y$ and an orthogonally quartic mapping $Q: X \rightarrow Y$ such that

$$
\|f(x)-A(x)-Q(x)\| \leq\left(\frac{1}{|2|\left(|2|-|2|^{p}\right)}+\frac{1}{|2|\left(|2|^{4}-|2|^{p}\right)}\right) \theta\|x\|^{p}
$$

for all $x \in X$.

Theorem 3.7. Let $0<p<1$. Let $f: X \rightarrow Y$ be a mapping satisfying $f(0)=0$ and (2.5). Then there exist an orthogonally additive mapping $A: X \rightarrow Y$ and an orthogonally quartic mapping $Q: X \rightarrow Y$ such that

$$
\|f(x)-A(x)-Q(x)\| \leq\left(\frac{1}{|2|\left(|2|^{p}-|2|\right)}+\frac{1}{|2|\left(|2|^{p}-|2|^{4}\right)}\right) \theta\|x\|^{p}
$$

for all $x \in X$.

\section{References}

[1] J. Alonso and C. Benítez, Orthogonality in normed linear spaces: a survey I. Main properties, Extracta Math. 3 (1988), 1-15.

[2] J. Alonso and C. Benítez, Orthogonality in normed linear spaces: a survey II. Relations between main orthogonalities, Extracta Math. 4 (1989), 121-131.

[3] G. Birkhoff, Orthogonality in linear metric spaces, Duke Math. J. 1 (1935), 169-172.

[4] S.O. Carlsson, Orthogonality in normed linear spaces, Ark. Mat. 4 (1962),297318.

[5] P.W. Cholewa, Remarks on the stability of functional equations, Aequationes Math. 27 (1984), 76-86.

[6] S. Czerwik, On the stability of the quadratic mapping in normed spaces, Abh. Math. Sem. Univ. Hamburg 62 (1992), 59-64.

[7] S. Czerwik, Functional Equations and Inequalities in Several Variables, World Scientific Publishing Company, New Jersey, London, Singapore and Hong Kong, 2002.

[8] S. Czerwik, Stability of Functional Equations of Ulam-Hyers-Rassias Type, Hadronic Press, Palm Harbor, Florida, 2003.

[9] D. Deses, On the representation of non-Archimedean objects, Topology Appl. 153 (2005), 774-785. 
[10] C.R. Diminnie, A new orthogonality relation for normed linear spaces, Math. Nachr. 114 (1983), 197-203.

[11] F. Drljević, On a functional which is quadratic on A-orthogonal vectors, Publ. Inst. Math. (Beograd) (N.S.) 54 (1986), 63-71.

[12] M. Fochi, Functional equations in A-orthogonal vectors, Aequationes Math. 38 (1989), 28-40.

[13] R. Ger and J. Sikorska, Stability of the orthogonal additivity, Bull. Pol. Acad. Sci. Math. 43 (1995), 143-151.

[14] S. Gudder and D. Strawther, Orthogonally additive and orthogonally increasing functions on vector spaces, Pacific J. Math. 58 (1975), 427-436.

[15] K. Hensel, Ubereine news Begrundung der Theorie der algebraischen Zahlen, Jahresber. Deutsch. Math.-Verein 6 (1897), 83-88.

[16] D.H. Hyers, On the stability of the linear functional equation, Proc. Natl. Acad. Sci. USA 27 (1941), 222-224.

[17] D.H. Hyers, G. Isac and Th.M. Rassias, Stability of Functional Equations in Several Variables, Birkhäuser, Basel, 1998.

[18] R.C. James, Orthogonality and linear functionals in normed linear spaces, Trans. Amer. Math. Soc. 61 (1947), 265-292.

[19] S. Jung, Hyers-Ulam-Rassias Stability of Functional Equations in Mathematical Analysis, Hadronic Press, Palm Harbor, Florida, 2001.

[20] A.K. Katsaras and A. Beoyiannis, Tensor products of non-Archimedean weighted spaces of continuous functions, Georgian Math. J. 6 (1999), 33-44.

[21] A. Khrennikov, Non-Archimedean analysis: quantum paradoxes, dynamical systems and biological models, Mathematics and its Applications 427, Kluwer Academic Publishers, Dordrecht, 1997.

[22] S. Lee, S. Im and I. Hwang, Quartic functional equations, J. Math. Anal. Appl. 307 (2005), 387-394.

[23] M.S. Moslehian, On the orthogonal stability of the Pexiderized quadratic equation, J. Difference Equ. Appl. 11 (2005), 999-1004.

[24] M.S. Moslehian, On the stability of the orthogonal Pexiderized Cauchy equation, J. Math. Anal. Appl. 318, (2006), 211-223.

[25] M.S. Moslehian and Th.M. Rassias, Orthogonal stability of additive type equations, Aequationes Math. 73 (2007), 249-259.

[26] M.S. Moslehian and Gh. Sadeghi, A Mazur-Ulam theorem in non-Archimedean normed spaces, Nonlinear Anal.-TMA 69 (2008), 3405-3408.

[27] P.J. Nyikos, On some non-Archimedean spaces of Alexandrof and Urysohn, Topology Appl. 91 (1999), 1-23.

[28] L. Paganoni and J. Rätz, Conditional function equations and orthogonal additivity, Aequationes Math. 50 (1995), 135-142.

[29] C. Park and J. Park, Generalized Hyers-Ulam stability of an Euler-Lagrange type additive mapping, J. Difference Equ. Appl. 12 (2006), 1277-1288.

[30] A.G. Pinsker, Sur une fonctionnelle dans l'espace de Hilbert, C. R. (Dokl.) Acad. Sci. URSS, n. Ser. 20 (1938), 411-414.

[31] Th.M. Rassias, On the stability of the linear mapping in Banach spaces, Proc. Amer. Math. Soc. 72 (1978), 297-300. 
[32] Th.M. Rassias, On the stability of the quadratic functional equation and its applications, Stud. Univ. Babeş-Bolyai Math. 43 (1998), 89-124.

[33] Th.M. Rassias, The problem of S.M. Ulam for approximately multiplicative mappings, J. Math. Anal. Appl. 246 (2000), 352-378.

[34] Th.M. Rassias, On the stability of functional equations in Banach spaces, J. Math. Anal. Appl. 251 (2000), 264-284.

[35] Th.M. Rassias (ed.), Functional Equations, Inequalities and Applications, Kluwer Academic Publishers, Dordrecht, Boston and London, 2003.

[36] J. Rätz, On orthogonally additive mappings, Aequationes Math. 28 (1985), 3549.

[37] J. Rätz and Gy. Szabó, On orthogonally additive mappings IV, Aequationes Math. 38 (1989), 73-85.

[38] F. Skof, Proprietà locali e approssimazione di operatori, Rend. Sem. Mat. Fis. Milano 53 (1983), 113-129.

[39] K. Sundaresan, Orthogonality and nonlinear functionals on Banach spaces, Proc. Amer. Math. Soc. 34 (1972), 187-190.

[40] Gy. Szabó, Sesquilinear-orthogonally quadratic mappings, Aequationes Math. 40 (1990), 190-200.

[41] S.M. Ulam, Problems in Modern Mathematics, Wiley, New York, 1960.

[42] F. Vajzović, Über das Funktional $H$ mit der Eigenschaft: $(x, y)=0 \Rightarrow H(x+$ $y)+H(x-y)=2 H(x)+2 H(y)$, Glasnik Mat. Ser. III 2 (22) (1967), 73-81.

Mathematics Branch

Seoul Science High School

Seoul 110-530, Korea

E-mail: 90pearl@naver.com

Mathematics Branch

Seoul Science High School

Seoul 110-530, Korea

E-mail: soshin6@naver.com

Mathematics Branch

Seoul Science High School

Seoul 110-530, Korea

E-mail: kguin96@naver.com

Mathematics Branch

Seoul Science High School

Seoul 110-530, Korea

E-mail: donghwan7401@naver.com 
Mathematics Branch

Seoul Science High School

Seoul 110-530, Korea

E-mail: bum2220@naver.com 